Rützel, Josef

\title{
Inklusion als Herausforderung für die beruflichen Schulen
}

Haushalt in Bildung \& Forschung 3 (2014) 1, S. 61-74

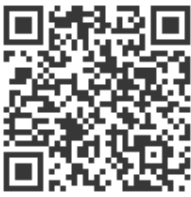

Quellenangabe/ Reference:

Rützel, Josef: Inklusion als Herausforderung für die beruflichen Schulen - In: Haushalt in Bildung \& Forschung 3 (2014) 1, S. 61-74 - URN: urn:nbn:de:0111-pedocs-204110 - DOI: 10.25656/01:20411

https://nbn-resolving.org/urn:nbn:de:0111-pedocs-204110

https://doi.org/10.25656/01:20411

in Kooperation mit / in cooperation with:

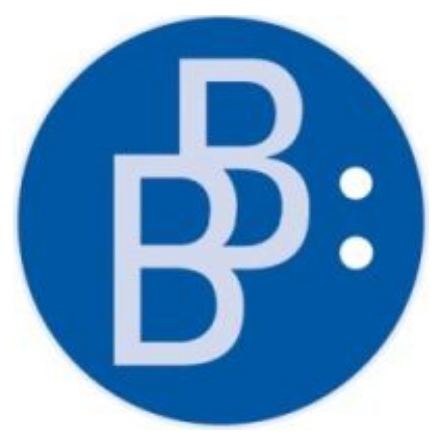

https://www.budrich.de

\section{Nutzungsbedingungen}

Gewährt wird ein nicht exklusives, nicht übertragbares, persönliches und beschränktes Recht auf Nutzung dieses Dokuments. Dieses Dokument ist ausschließlich für den persönlichen, nicht-kommerziellen Gebrauch bestimmt. Die Nutzung stellt keine Übertragung des Eigentumsrechts an diesem Dokument dar und gilt vorbehaltlich der folgenden Einschränkungen: Auf sämtlichen Kopien dieses Dokuments müssen alle Urheberrechtshinweise und sonstigen Hinweise auf gesetzlichen Schutz beibehalten werden. Sie dürfen dieses Dokument nicht in irgendeiner Weise abändern, noch dürfen Sie dieses Dokument für öffentliche oder kommerzielle Zwecke vervielfältigen, öffentlich ausstellen, aufführen, vertreiben oder anderweitig nutzen

Mit der Verwendung dieses Dokuments erkennen Sie die Nutzungsbedingungen an.

\section{Terms of use}

We grant a non-exclusive, non-transferable, individual and limited right to using this document.

This document is solely intended for your personal, non-commercial use. Use of this document does not include any transfer of property rights and it is conditional to the following limitations: All of the copies of this documents must retain all copyright information and other information regarding legal protection. You are not allowed to alter this document in any way, to copy it for public or commercial purposes, to exhibit the document in public, to perform, distribute or otherwise use the document in public.

By using this particular document, you accept the above-stated conditions of use.

\section{Kontakt / Contact:}

\section{peDOCS}

DIPF | Leibniz-Institut für Bildungsforschung und Bildungsinformation Informationszentrum (IZ) Bildung

E-Mail: pedocs@dipf.de

Internet: www.pedocs.de

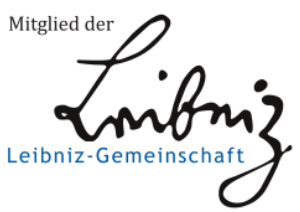


3. Jahrgang Heft 1

2014

产

m

$\bullet$

$\frac{0}{2}$

$\diamond$

ธุ

\$্ণ

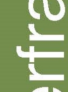

$\Phi$

$\frac{\mathrm{c}}{\mathrm{C}}$

징

인

$\stackrel{\frac{2}{亠}}{>}$

$\diamond$

$\stackrel{c}{\Phi}$

$\frac{\mathrm{O}}{\mathrm{d}}$

$\frac{\Phi}{C}$

$\bar{\Phi}$

E

E

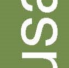

$\stackrel{\text { N }}{s}$

$\diamond$

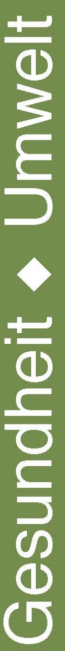

ISSN 2193-8806
Haushalt in

Forschung

\section{Schwerpunktthemen:}

Berufsdidaktische Forschung Diversität und Inklusion

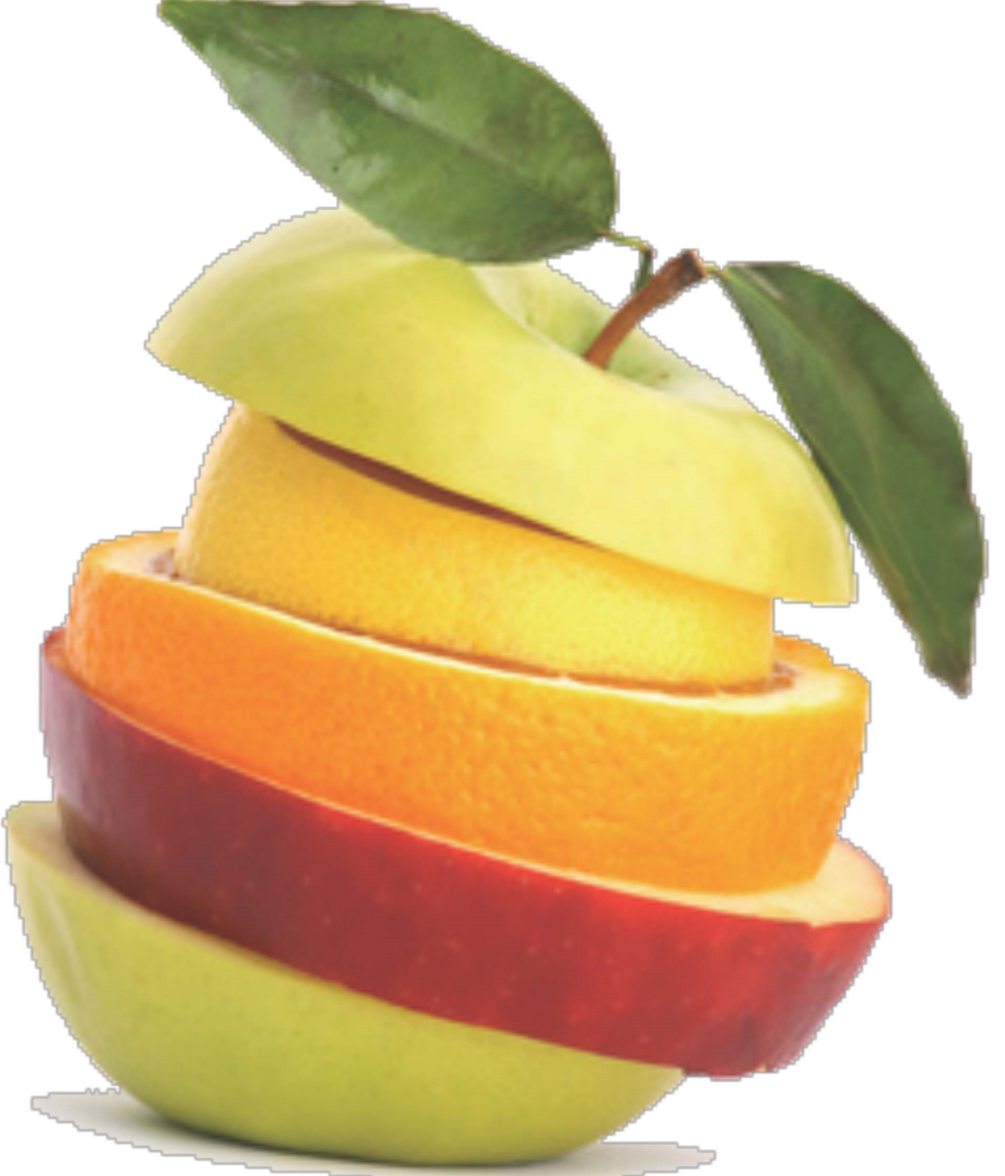

Verlag Barbara Budrich 
Inhaltsverzeichnis |

Irmhild Kettschau

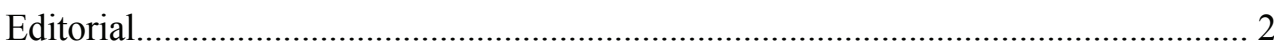

Kathrin Gemballa-Witych

Didaktisch intendierte Forschung im Berufsfeld Ernährung und Hauswirtschaft....... 3

Stephan Stomporowski

Die Bedeutung ,Praktischen Wissens` für die Entwicklung gestaltungsorientierter

Curricula

Nicole Riemer

Lernsituation „Was hat der Regenwald mit mir/mit uns zu tun?“ im Spiegel des

Angebots-Nutzungsmodells nach Helmke.

Doris Wisse

Der Konditorberuf im Spannungsfeld von handwerklicher Tradition und modernen Marktmechanismen

Josef Rützel

Inklusion als Herausforderung für die beruflichen Schulen. 61

Christine Keller und Thies Rohmann

Inklusion - Umsetzung in den Bundesländern

Mona Massumi

Diversität in der Lehrerinnen- und Lehrerbildung - zur Bedeutung von

Lehrkräften mit Migrationshintergrund.

Alexandra Brutzer

Niederschwelligkeit in personenbezogenen Dienstleistungsberufen. 96

Julia Kastrup

Rezension: Nachhaltigkeit im Berufsfeld Ernährung und Hauswirtschaft. 107

Kathrin Gemballa-Witych

Rezension: G + Einladung zum Leichenschmaus für das Gastgewerbe

Irmhild Kettschau

Bundesarbeitsgemeinschaft für Berufsbildung in der Fachrichtung Ernährung und Hauswirtschaft.

Arbeits- und Studienbuch Sensorische Prüfungen 
Inklusion als Herausforderung |

Josef Rützel

\section{Inklusion als Herausforderung für die beruflichen Schulen}

In diesem Beitrag geht es darum, Herausforderungen an die berufliche Bildung und die beruflichen Schulen zu thematisieren, die unter den Bedingungen der gesellschaftlichen Transformationsprozesse durch Inklusion entstehen. Hierzu werden das Verständnis von Inklusion und inklusiver Bildung sowie die Effekte von inklusiver Bildung reflektiert. Darüber hinaus Wege zur Inklusion aufgezeigt.

Schlüsselwörter: Inklusion, inklusive Bildung, Effekte von Inklusion, demographischer Wandel, Berufsschulentwicklung

\section{Ausgangslage und Hintergrund}

Das Thema Inklusion ist hochaktuell und prägt viele Diskussionen. Besonders die am 03.Mai 2008 in Kraft getretene UN Konvention über die Rechte von Menschen mit Behinderungen (nachfolgend UN-BRK) gab Impulse für die Inklusionsdebatte. In der UN-BRK werden für alle Lebensbereiche Ziele formuliert, um die Partizipation von Menschen mit Behinderungen zu erleichtern und um Diskriminierung und Ausschluss zu vermeiden. Es gibt keine konkreten Vorgaben zur Umsetzung dieser Ziele. Verpflichtend sind jedoch eine überprüfende Begleitung (Monitoring) und eine regelmäßige Berichtspflicht.

Die Bundesrepublik Deutschland unterzeichnete die UN-BRK im Februar 2009. Sie ist seit 26. März 2009 gültig. Im Juni 2011 verabschiedete die Bundesregierung einen nationalen Aktionsplan in welchem sie sich verpflichtete, zur Umsetzung dieser Konvention eine Strategie aus Zielen und Maßnahmen zu entwickeln. Das Institut für Menschenrechte in Berlin wurde mit der Durchführung des Monitoring beauftragt (Aichele, 2010). Als erstes Bundesland veröffentlichte die Landesregierung von Rheinland-Pfalz bereits am 26.03.2010 einen Aktionsplan zur Umsetzung der UNBRK. Inzwischen haben insgesamt zehn Bundesländer Aktionspläne zur Inklusion verabschiedet (Aichele, 2010).

Da sich die UN-BRK und die Aktionspläne der Bundes- und Landesregierungen auf alle Lebensbereiche beziehen, gibt es ebenfalls Aktionspläne, Beschlüsse und Initiativen für den Bildungs-, Ausbildungs- und Beschäftigungsbereich. Die Zahl der Aktionspläne, Initiativen und der Stellungnahmen dazu ist kaum noch überschaubar. Erwähnt werden sollen hier lediglich der Beschluss der Kultusministerkonferenz 


\section{Inklusion als Herausforderung}

(KMK) „Inklusive Bildung von Kindern und Jugendlichen“ in Schulen vom 20.10.2011 sowie die Inklusionsinitiative für Ausbildung und Beschäftigung, die Teil der Umsetzung des Nationalen Aktionsplans ist (Bundesministerium für Arbeit und Soziales (BAMS), 2013a). Aufmerksam zu machen ist auch auf die teilweise sehr umfangreichen Gutachten, die im Kontext dieser Debatte in Auftrag gegeben wurden (vgl. u.a. Klemm \& Preuss-Lausitz, 2011).

In der schulischen Praxis und insbesondere in der Praxis der beruflichen Schulen gibt es bisher jedoch nur erste Schritte hin zur Inklusion. Hierfür sind mehrere Gründe zu nennen. Die Debatte insgesamt ist noch recht jung und das Thema Inklusion ist hochkomplex. Um ein inklusives Bildungssystem zu entwickeln sind Veränderungen auf allen Ebenen des Bildungssystems erforderlich: Auf der staatlichen Ebene durch neue (gesetzliche) Rahmenbedingungen und neue Steuerungs- und Ordnungsinstrumente, auf der regionalen Ebene durch ,inklusive“ Bildungsentwicklungspläne, die Vernetzung der Institutionen und Akteure, die regionale Steuerungsgruppen etc.. Schließlich sind auf der Ebene der Einzelschule eine höhere Autonomie und Gestaltungsspielräume hinsichtlich der Angebote und der Angebotsorganisation, der Rekrutierung des Bildungspersonals erforderlich. Ein inklusives Schulleitbild muss entwickelt, eine inklusive Schulkultur geschaffen werden. Auf der Unterrichtsebene erfordert das Ziel der Inklusion die Verwirklichung der Merkmale guten Unterrichts, bezogen auf die Gegenstände, die zeitlichen Abläufe, die Bildung von Lerngruppen etc., neue Differenzierungsformen und neue Formen des kooperativen Lernens.

Das Thema Inklusion ist aber auch hoch emotional geprägt. Eltern von leistungsstarken Jugendlichen und leistungsstarke erwachsene Lerner selbst befürchten, dass sie in ihrem Lernen behindert werden und nicht optimal ihre Potentiale entfalten können bzw. dass das Leistungsniveau in Inklusionsgruppen sinkt. Eltern von Jugendlichen mit Behinderungen als auch Erwachsene mit Behinderung befürchten, dass sie in Inklusionsgruppen leiden, dass ihnen der Schutzraum und die Zuwendung für die besondere Förderung abhandenkommen. Ein hohes Hindernis sind weiter die Barrieren, die im Beharrungsvermögen von Strukturen sowie der Menschen die in diesen Strukturen arbeiten, denken und handeln, bestehen. Es fehlen Unterstützungssysteme, Modelle zur Veränderung der Strukturen, es fehlt an Professionalität, Differenzierungskonzepten und insbesondere an unserer Fähigkeit, eingefahrene Routinen und Deutungsmuster zu überwinden. Barrieren gibt es vor allem in unseren Köpfen. Die Potentiale von Vielfalt und Diversität sind oft nicht unmittelbar erkennbar. Das, was wahrgenommen wird, sind die zu überwindenden Widerstände und die damit verbundenen Anstrengungen.

\section{Zum Verständnis von Inklusion}

Inklusion ist keineswegs, wie es zunächst den Anschein hat, ein klar definierter und eindeutiger Begriff (vgl. Tenorth, 2013). Zum einen ist die Differenz zwischen In- 


\section{Inklusion als Herausforderung |}

klusion und Integration nicht immer deutlich und zum anderen lassen sich in der Debatte zur Inklusion ein enger und ein weiter Begriff von Inklusion ausmachen.

In der Sonderpädagogik wird Inklusion als erweiterte und ,optimierte Integration" gesehen: Integration beinhaltet demnach eine sonderpädagogische Unterstützung für spezifische Zielgruppen, während Inklusion alle mit ihren jeweils spezifischen pädagogischen Bedürfnissen in den Blick nimmt. Das Inklusionskonzept wird als Theorie einer pädagogisch nicht unterteilbaren heterogenen Gruppe beschrieben, wonach gemeinsames, aber individuelles Lernen und ein individualisiertes Curriculum für alle vorgesehen sind. Bedeutend ist, dass im Inklusionskonzept Menschen mit Behinderungen oder Benachteiligungen nicht mehr als eine eigene „förderungsbedürftige“ abgegrenzte Gruppe betrachtet werden (vgl. Bylinski \& Rützel, 2011, S. $15)$.

Wie aus der Bezeichnung ersichtlich, zielt die UN-BRK auf die Gruppe der Menschen mit Behinderung. Auf diese Gruppe beziehen sich auch die Aktionspläne, Beschlüsse und Initiativen. So wird zum Beispiel vom Bundesministerium für Arbeit und Soziales (BAMS) als Handlungsperspektive hervorgehoben:

(...) Menschen mit Behinderungen eine gleichberechtigte Teilhabe am politischen, gesellschaftlichen, wirtschaftlichen und kulturellen Leben zu ermöglichen, Chancengleichheit in der Bildung und in der Arbeitswelt herzustellen und allen Bürgerinnen und Bürgern die Möglichkeit auf einen selbstbestimmten Platz in einer barrierefreien Gesellschaft zu geben. (BAMS, 2013a)

Für Viele greift jedoch die Eingrenzung der Inklusionsdebatte und der Bemühungen um Inklusion auf Menschen mit Behinderung zu kurz. Unstrittig ist, dass diese Diskussion den Blick auf Menschen mit Behinderung verändert hat. Deren Teilhabe wird in diesem Verständnis nicht durch die Beeinträchtigung, sondern durch soziale und gesellschaftliche Strukturen behindert. Das gilt auch für die Barrieren im Bildungssystem. Behinderung ist folglich ein relationaler Begriff, denn individuelle Dispositionen treffen auf gesellschaftliche Bedingungen von „Normalität“ die Barrieren und Behinderungen erzeugen. Gleichwohl wird der Gleichheitsgrundsatz des Grundgesetzes in der Inklusionsdebatte nur auf die Gruppe der Menschen mit Behinderung fokussiert. Andere marginalisierte oder von Marginalisierung bedrohte Gruppen wie Menschen mit Migrationshintergrund, schulische Außenseiter, Arme, sozial Randständige, Homo- und Transsexuelle, alte Menschen etc. werden in dieser Debatte bisher nicht berücksichtigt. Nach dem internationalen Referenzdokument der 48 . UNESCO Weltkonferenz der Bildungsminister in Genf im Jahre 2008 geht Inklusion jedoch eindeutig über die Eingliederung von Menschen mit Behinderung in das Regelsystem hinaus (vgl. Sicking, 2012, S. 4ff).

Doch auch die Zielsetzung, möglichst alle potenziell marginalisierten Gruppen bei der Weiterentwicklung des Bildungssystems zu berücksichtigen, greift letztlich zu kurz. Vielmehr geht es darum, auf Etikettierung und Segmentierung gänzlich zu verzichten und allen vorbehaltlos auf der Basis ihrer individuellen Kompetenzen, Potenziale und Bedürfnisse eine optimale Bildung zu ermöglichen (vgl. a.a.O., S. 5). 


\section{Inklusion als Herausforderung}

Erst in diesem Verständnis kann inklusive Bildung als ein Menschenrecht umgesetzt werden.

Inklusion betont nach diesem Verständnis der Deutschen UNESCO-Kommission (2009) den im Artikel 3 des Grundgesetzes formulierten Gleichheitsgrundsatz für alle Menschen. Danach zielt Inklusion auf die gesellschaftliche Teilhabe aller Menschen unabhängig von ihren individuellen Dispositionen.

Inklusive Bildung nimmt in diesem Verständnis vorbehaltlos jeden Menschen in seiner Einzigartigkeit und seinen individuellen Bedürfnissen in den Blick. Sie steht für gleichberechtigte Teilhabe an den Bildungsangeboten für alle Menschen zur Entfaltung und Entwicklung der eigenen Möglichkeiten und für mehr Chancengleichheit durch Vielfalt. Inklusive Bildung stellt die unterschiedlichen Bedürfnisse und Voraussetzungen der Lernenden in den Mittelpunkt. Das (Berufs-) Bildungssystem wird an die Bedürfnisse der Lernenden angepasst und nicht umgekehrt. Sie orientiert sich an Bildungsgerechtigkeit und Chancengleichheit als ethisch-moralische Verpflichtung.

\section{Zur Wirksamkeit inklusiver Bildung}

Strittig ist in der Debatte, ob die durch Inklusion erwarteten besseren Bildungsergebnisse auch tatsächlich erzielt werden. Bisher liegen für den Bereich der allgemeinbildenden Schulen vor allem international einige Studien vor (vgl. zusammenfassend Möller, 2013). Zur Systematisierung der Befunde unterscheidet er in Effekte der Inklusion auf schulische Leistungen und auf psychosoziale Variablen der Schülerinnen und Schüler mit und ohne sonderpädagogischem Förderbedarf. Dabei ist die Vergleichbarkeit der sich auf verschiedene Länder beziehenden Studien eingeschränkt, denn wichtige Faktoren wie die Ausgestaltung der Inklusionsansätze, das Verständnis von Behinderung bzw. von Förderbedarf und deren Diagnostik sowie die gesellschaftliche Stellung der Menschen mit Behinderungen variieren sehr stark. Zudem betont Möller (2013, S. 15f), dass er den Fokus ausschließlich auf die schulischen Leistungen legt.

Zusammengefasst zeigen die Studien geringe positive Effekte auf die schulischen Leistungen der inklusiv beschulten Schülerinnen und Schüler mit sonderpädagogischem Förderbedarf. Die Befunde beziehen sich zudem vorwiegend auf Schülerinnen und Schüler mit leichten kognitiven Einschränkungen aus dem Förderschwerpunkt Lernen (vgl. Möller, 2013, S. 24). Hinsichtlich der psychosozialen Variablen zeigen sich sehr schwache negative Wirkungen der inklusiv beschulten Schülerinnen und Schüler mit sonderpädagogischem Förderbedarf auf das schulische Selbstkonzept. Die Befunde zu anderen Variablen wie die soziale Integration sind nicht eindeutig (vgl. Möller, 2013, S. 27). Hinsichtlich der schulischen Leistungen der Schülerinnen und Schüler ohne sonderpädagogischen Förderbedarf gibt es kein klares Fazit, jedoch können negative Effekte nicht belegt werden. 


\section{Inklusion als Herausforderung |}

Klemm und Preuss-Lausitz (2011) referieren auch Studien, die neben den unmittelbaren schulischen Leistungen auch die Wirkungen auf die weitere Biographie untersuchen. Demnach scheint belegt, dass gemeinsamer Unterricht für Kinder mit Förderbedarf in leistungsgemischten Klassen lerneffektiver ist als in Behinderungshomogenen Klassen. Für die Kinder ohne Förderbedarf bleiben die kognitiven Leistungen etwa gleich. Bei beiden Gruppen werden die sozialen Kompetenzen, wie realistische Selbsteinschätzungen, Selbstsicherheit und Selbstverantwortung gestärkt und die sozialen Beziehungen auch außerhalb des Unterrichts gefördert (vgl. Klemm \& Preuss-Lausitz, 2011, S. 36).

Darüber hinaus hat gemeinsamer Unterricht langfristig positive Wirkung auf die berufliche und soziale Teilhabe und die Lebensgestaltung insgesamt. Referiert werden Ergebnisse von Langzeitstudien aus Norwegen, der Schweiz und den USA. Besonders bemerkenswert sind die Ergebnisse aus Norwegen und der Schweiz. In Norwegen waren Kinder und Jugendliche, die inklusiv beschult wurden deutlich erfolgreicher als die aus Sonderklassen. Rund 60\% erreichten das Kompetenzniveau der Sekundarstufe II, dagegen nur 35\% derjenigen aus Sonderklassen (vgl. Klemm \& Preuss-Lausitz, 2011, S. 37f).

Studien aus der Schweiz haben darüber hinaus gezeigt, dass Jugendliche aus Inklusionsklassen signifikant häufiger eine Lehre absolvieren und eine Beschäftigung erreichen, die ihrer Ausbildung entspricht. Darüber hinaus zeigte sich noch mehrere Jahre nach dem Schulabschluss ein positiver Einfluss auf das Anspruchsniveau in der Berufssituation sowie langfristige positive Einflüsse auf die Einschätzung der eigenen Fähigkeiten und auf die Stabilität der privaten Beziehungen (vgl. Klemm \& Preuss-Lausitz, 2011, S. 38ff).

\section{Gesellschaftliche Transformationsprozesse}

Inklusion wird hier als Möglichkeit der gesellschaftlichen Teilhabe unter den Bedingungen einer sich in Transformation befindlichen Gesellschaft verstanden. In unserer heutigen Transformationsgesellschaft ist der Wandel die Normalität. Wir leben in einer globalisierten, individualisierten Medien- und Wissensgesellschaft, so die Schlagworte, die vielfältig thematisiert und beschrieben sind (vgl. u.a. Rützel, 2000, S. 27ff). Diese weitreichenden Entwicklungen bedingen und durchdringen sich gegenseitig. Lebensräume werden zunehmend internationalisiert und mediatisiert, wie die Erwerbsarbeit, die Schule und das gesamte Privatleben. Alleine die Entwicklung von PCs, Smartphones oder Tablets kann eine Vorstellung davon bieten, wie sich Alltag, Bildung oder Arbeit verändert haben und weiter verändern werden. Die Reichweite des Einsatzes der Mikrotechnologie in der Produktion, im Dienstleistungs- und Gesundheitsbereich, in der Forschung und Entwicklung können die meisten von uns nur erahnen. 


\section{Inklusion als Herausforderung}

Außerdem haben die Medien und Technologien einen hohen Anteil am Wissenszuwachs, -zerfall und am Zugang zu Wissen. Wissenszuwachs und -zerfall unterstützen die massiven Wandlungsprozesse der bestehenden Strukturen, Disziplinen und Kulturen. Das Zurückdrängen des Produktionsparadigmas, die Zunahme des Dienstleistungssektors und von Forschung und Entwicklung sind ebenso Folgen wie Veränderungen im für die Berufspädagogik grundlegenden Verhältnis von Wissen und Erfahrung.

Die durch diese Entwicklungen fortschreitende Endstandardisierung der Biographien und Lebenslagen führt zur Zunahme der Vielfalt in allen Altersgruppen und zu höheren Anforderungen an die Biographiegestaltung. Die Vielfalt der Lebenskonzepte, -lagen, individuellen Voraussetzungen und Interessen nimmt insgesamt, insbesondere aber auch bei Älteren erheblich zu.

Vor dem Hintergrund der technologischen Entwicklung ist zudem von dem Begriff „Mensch 2.0“ die Rede. Dieser ist nach Damberger (2014) ein Doppelgeschöpf, welches sowohl in der realen als auch der virtuellen Welt Zuhause ist. Zugleich wird durch die Verbindung von Mensch und Computer eine erweiterte Realität geschaffen, bei der die Trennung zwischen realer und virtueller Welt mehr und mehr aufgelöst wird. Der „Mensch 2.0“ dehnt sich nicht nur im virtuellen Raum aus, sondern verbindet tendenziell das Reale mit dem Virtuellen zu einem Hyperraum.

\section{Demographischer Wandel}

Ein wesentlicher Bestandteil der gesellschaftlichen Transformation ist der demographische Wandel. Inzwischen wird er auch in der Berufspädagogik intensiv diskutiert, weil die damit verbundenen Folgen und Herausforderungen ganz offensichtlich und auf allen Ebenen des (Berufs-) Bildungssystems spürbar sind.

In den Bevölkerungsprognosen lassen sich einige wesentliche Trends konstatieren: die Gesamtbevölkerung nimmt ab, die Abnahme trifft die ländlichen Regionen, in den Ballungszentren steigt die Bevölkerung; die Bevölkerung wird wegen der steigenden Lebenserwartung deutlich älter, vor allem der Anteil und die Zahl der über 60jährigen nimmt merklich zu; und der Anteil der Menschen mit Migrationshintergrund vergrößert sich, auch bei den Älteren.

Die mit den größten Unsicherheiten behaftete Einflussgröße in den Bevölkerungsprognosen ist der Wanderungssaldo. In den Vorausberechnungen des Statistischen Bundesamts wird der jährliche Wanderungssaldo nach verschiedenen Modellannahmen in einer Größenordnung zwischen 100.000 und 200.000 angesetzt (vgl. Statistisches Bundesamt, 2009, S. 33). In 2011 betrug jedoch der reale Wanderungssaldo 279.000 und in 2012 betrug er 369.000. In den nächsten Jahren wird eher noch ein weiterer Anstieg erwartet. Die Annahmen in den Prognosen liegen folglich deutlich unter dem gegenwärtigen und in nächster Zeit erwarteten Wanderungssaldo. 


\section{Inklusion als Herausforderung |}

Angesprochen ist damit auch der Anteil der Bevölkerung mit Migrationshintergrund. Besonders der Anteil der jüngeren Menschen mit Migrationshintergrund wird weiter zunehmen. 2010 hatten 19\% der Bevölkerung einen Migrationshintergrund. Deren Anteil beträgt aktuell bei den 24-jährigen ca. $23 \%$ und bei den einjährigen bereits 35\% (vgl. Autorengruppe Bildungsberichterstattung, 2012, S. 17). Derzeit sind jedoch lediglich 45\% der Personen mit Migrationshintergrund Ausländerinnen und Ausländer. Zu beobachten ist, dass die Anzahl der Länder, aus denen die Migranten zuwandern, sich vergrößert. Daraus kann geschlossen werden, dass sich durch die Migration die Vielfalt erheblich erhöht. Neben der Vielfalt der Nationalitäten, der Migrationsgeschichte und des „Migrationsstatus“ führt vor allem die unterschiedliche Bewältigung der Migrationsbedingungen zur Vervielfältigung der Lebenskonzepte.

Diese allgemeinen Entwicklungstrends erfahren nach Bundesländern und Regionen sehr unterschiedliche Ausprägungen. Sowohl die Abnahme der Bevölkerung, als auch die Alterspyramide unterscheidet sich in den einzelnen Bundesländern und Regionen erheblich. In manchen Regionen und Ballungszentren ist sogar ein $\mathrm{Zu}-$ wachs zu verzeichnen, während in anderen Regionen die Abnahme der Einwohnerzahlen und die Alterspyramide erheblich über dem Durchschnitt liegen.

\section{Fachkräftemangel}

Diskutiert wird der demographische Wandel insbesondere im Hinblick auf seine Auswirkungen auf den Fachkräftebedarf, den Arbeitsmarkt und die Auswirkungen auf das Berufsbildungssystem. Durch den demographischen Wandel sinkt die Zahl der Personen im erwerbsfähigen Alter. Dadurch wird mittelfristig ein Fachkräftemangel erwartet (Gans, 2011, S. 109). Gleichzeitig wird von der Fortsetzung des Trends zur Höherqualifizierung ausgegangen (vgl. Autorengruppe Bildungsberichterstattung, 2010, S. 161ff). Der Fachkräftemangel ist nicht flächendeckend und in allen Berufsfeldern bzw. Berufsgruppen spürbar. Mangel herrscht insbesondere an Experten und Fachkräften der Maschinen- und Fahrzeugtechnik, der Mechatronik, der Elektro- und Energietechnik, sowie im Bereich der Informatik, Softwareentwicklung und IT-Beratung. Fachkräfte fehlen in der Gesundheits-, Kranken- und Altenpflege, in der Gastronomie und den Reinigungsberufen, in der Sanitär-, Heizungsund Klimatechnik, sowie bei den Verkehrs-, Lager-, Transport-, Sicherheits- und Wachberufen (vgl. Baethge, 2012; Sarter, 2013).

Das Reservoir zur Erhöhung des Fachkräftepotenzials bilden drei Gruppen:

(1) Die Jugendlichen im Übergangssystem, die durch besondere Förderung und Unterstützungsmaßnahmen zu einem Ausbildungsabschluss geführt werden sollen. Dazu gibt es von zahlreichen öffentlichen Institutionen auf Bundes- und Landesebene sowie von Sozialpartnern und Verbänden 


\section{Inklusion als Herausforderung}

Aktionsprogramme, neue Konzepte und Initiativen. Beispielhaft zu nennen sind: „Perspektive Berufsabschluss“, „BMBF - Bildungsketten Initiative“, Konzept „Neues Übergangssystem Schule-Beruf NRW“, Bertelsmann-Initiative „Chance Ausbildung“.

(2) Die Anwerbung von Fachkräften aus anderen Ländern: diese richtet sich nicht mehr nur auf Führungskräfte, sondern auch auf Personen auf Facharbeiterniveau und auf Auszubildende. Hier gibt es Initiativen des Bundes und auf Länder-, Bezirks- und Kammerebene. Unter anderem gibt es auch Projekte zu Gewinnung von Fachkräften in ländlichen Regionen.

(3) Die ältere Generation, die erst allmählich als Arbeitskräftepotential wahrgenommen wird und deren Erfahrung, Kompetenzen und Begabungen durch Bildung mobilisiert werden sollen (vgl. Gans, 2011, S. 52ff). Dabei geht es nicht nur um die Aufrechterhaltung des Arbeitsvermögens, vielmehr ist Bildung auch im Alter eine wichtige Voraussetzung für Teilhabe und eine selbstbestimmte Lebensführung. Die Teilnahme an Bildung wirkt sich positiv auf die Lebenszufriedenheit und Gesundheit aus (vgl. Ahmadi \& Kolland, 2010, S. 43), sie leistet zudem einen entscheidenden Beitrag zur Aufrechterhaltung kognitiver, physischer und psychischer Fähigkeiten und trägt damit zur Wahrung eines autonomen Lebensstils bis ins hohe Alter bei (vgl. Tippelt, Schmidt, Schnurr, Sinner $\&$ Theisen, 2009, S. 15). Eine für das Thema Inklusion besonders wichtige Entwicklung ist, dass auch im Alter die Vielfalt der Lebenskonzepte, der Erfahrungen, der Lebenslagen erheblich zugenommen hat und weiter zunimmt.

\section{Herausforderungen und Lösungswege für die berufliche Bildung}

Die Auswirkungen der aufgezeigten Trends und Entwicklungen auf das Berufsbildungssystem und die beruflichen Schulen sind erheblich. Aus demographischer Perspektive wirkt sich vor allem der Rückgang der unter 24jährigen aus, die besonders in Flächenländern zu spüren sind. So ist beispielsweise in Rheinlandpfalz die Zahl der Schülerinnen und Schüler an öffentlichen Berufsschulen von 124.000 im Schuljahr 2008/09 auf 118.000 in 2011/12 gesunken. Rückgänge sind auch in andern Schulformen zu verzeichnen, etwa in den Berufsfachschulen und im Berufsvorbereitungsjahr. Quantitativ besonders stark werden in den ländlichen Regionen die Schülerzahlen in der dualen Ausbildung zurückgehen. Gestiegen sind dagegen die Schülerzahlen im Beruflichen Gymnasium und in den Fachschulen (Expertengruppe zur Weiterentwicklung der beruflichen Schulen in Rheinland-Pfalz, 2013). 


\section{Inklusion als Herausforderung}

Unter Beibehaltung der jetzigen Strukturen könnte es unter den geschilderten Bedingungen rein rechnerisch zu Klassengrößen von teilweise unter drei Schülern kommen. In Rheinland-Pfalz liegt der Anteil der Klassengrößen kleiner als 16 bei fast einem Drittel. Knapp 7\% der Klassen haben weniger als 8 Schülerinnen und Schüler (Expertengruppe zur Weiterentwicklung der beruflichen Schulen in Rheinland-Pfalz, 2013). Die Folge wäre, dass ganze Bildungsgänge und Ausbildungsangebote geschlossen werden müssten und Schulen in ihrem Bestand bedroht wären. Dies würde dazu führen, dass es in bestimmten Regionen keine Berufsschulen mehr gäbe. Notwendig wäre dann die Konzentration von Angeboten z.B. durch landes- oder bundesweite Fachklassen. In Kauf genommen werden müssten dabei hohe öffentliche Kosten, unter anderem für Transport oder Unterbringung. Eine wohnortnahe Bildungsversorgung wäre dann nicht mehr gegeben, mit negativen Folgen für die wirtschaftlichen, sozialen und kulturellen Strukturen in den Regionen, in denen die Angebote eingestellt werden.

Auf der anderen Seite steigen die Bildungsbedarfe erheblich. Steigende Bildungsbedarfe gibt es in der Ausbildung der Gruppe der Benachteiligten, aber auch durch Zusatzangebote für leistungsstarke Schülerinnen und Schüler. Die gesellschaftlichen Megatrends wie Technologisierung, Wissenszuwachs und Internationalisierung rufen einen steigenden Bedarf an Weiterbildung hervor. Ebenso bedürfen im Ausland angeworbene Fachkräfte zusätzlicher Bildungsangebote da ihnen eventuell für den deutschen Arbeitsmarkt erforderliche Kompetenzen fehlen. Und schließlich wird auch die Aufrechterhaltung der Beschäftigungsfähigkeit älterer Arbeitnehmerinnen und Arbeitnehmer angepasste, neue Bildungsangebote für diese Gruppe erfordern.

Inklusion, verbunden mit regionaler Vernetzung und Koordination, schulischer Autonomie und flexibler curricularer Angebotsgestaltung, bietet hier enormes Lösungspotenzial. Wie eingangs erläutert, wird im weiten Begriff von inklusiver Bildung vorbehaltlos jeder einzelne Mensch in seiner Einzigartigkeit und seinen individuellen Bedürfnissen in den Blick genommen. Inklusive Bildung stellt die individuellen Bedürfnisse und Voraussetzungen der Lernenden in den Mittelpunkt und passt das (Berufs-)Bildungssystem an die Bedürfnisse der Lernenden an. Bildungsgerechtigkeit und Chancengleichheit sind ethisch-moralische Verpflichtungen unserer Gesellschaft. Aus Vielfalt ergibt sich in diesem Sinne Potential für Chancengleichheit.

Barrierefreiheit und die Philosophie des Universellen Designs (vgl. Rützel, 2013) sind Orientierungspunkte für alle Ebenen, Strukturen, Organisationsformen des Bildungssystems.

Universal Design meint weder Standardisierung noch kulturelle Uniformität. Dem Konzept des Universal Design liegt ein sozialer, ein am Menschen orientierter Gestaltungsansatz zugrunde, der zum Ziel hat, die gesamte von Menschen für Menschen gestaltete Umwelt für möglichst viele zugänglich und nutzbar zu machen. Ungeachtet ihrer individuellen Fähigkeiten, ihre Alters und Geschlechts oder ihres 


\section{Inklusion als Herausforderung}

kulturellen Hintergrunds soll allen Menschen eine gleichberechtigte Teilhabe an der Gesellschaft ermöglicht werden. Stigmatisierung durch eine Gestaltung, die Menschen von der Inanspruchnahme und Nutzung bestimmter Dienstleistungen, Räume und Produkte ausschließt, soll von vorneherein vermieden werden. (Internationales Design Zentrum (IDZ), 2014)

Barrierefreiheit wird im Kontext des Bildungssystems und der Beruflichen Bildung bezogen auf die Struktur des Bildungssystems, die Übergänge, die regionale Angebotsstruktur, die Organisation der Bildungseinrichtung, die Curricula, die Lehr/Lernformen, die Lernmaterialien, u.a. barrierefreies E-Learning (vgl. Fisseler \& Schaten, 2012), die Schulgebäude und die Ausstattung thematisiert.

Generell lässt sich konstatieren, dass die Wege zur Inklusion anschlussfähig sind an die Debatten zur Differenzierung und Flexibilisierung des (Berufs-) Bildungssystems, zu regionalen Netzwerken, zur Organisations- und Qualitätsentwicklung von Schule, zum guten Unterricht und zur Professionalisierung des Lehrpersonals. Auf allen Ebenen geht Inklusion aber auch über diese Ansätze hinaus. Für alle Ebenen gibt es aber auch Handlungsansätze.

Abgestimmte Inklusionspläne sind auf der Landesebene und auf den Ebenen der Region und der Einzelschulen zu entwickeln und festzuschreiben. Auf der Landesebene sind die entsprechenden Gesetze, die Verordnungen und die Steuerungsinstrumente zu prüfen und zu verändern. Dazu gehören die Vorgaben zu Klassengrößen, zur Autonomie der Schulen, zu Bildung regionaler Netzwerke und Steuerungsgruppen, sowie neue Formen des Monitoring. Auf der regionalen und lokalen Ebene sind neue Ansätze der Bildungsplanung und der regionalen Steuerung auf der Basis von Inklusionsprinzipien notwendig. An diesen sind die regionalen Bildungsakteure zu beteiligen. Zur Unterstützung der regionalen Inklusion hat Reich (2012, S. 34-51) ein verbindliches Leitbild für Inklusion und Bildungsgerechtigkeit ausgearbeitet, welches zehn Verpflichtungen und fünf Standards enthält. Dieses Leitbild mit seinen Standards und Verpflichtungen kann die konkrete Entwicklung und Umsetzung orientieren, unterstützen und überprüfbar machen. Darüber hinaus hat Patt (2012, S. 210f) 13 Gütekriterien für die Kommunale Inklusionsplanung ausgearbeitet. Viele Vorschläge enthält auch das Gutachten von Klemm und PreussLausitz (2011, S. 97ff).

Wenn eine Beschulung nahe zum Wohnort bzw. zur Ausbildungsstätte gesichert werden soll, sind aufgrund der demographischen Entwicklung regionale und insbesondere schulspezifische Steuerungen der Klassen und Lerngruppenbildung, die Flexibilisierung der Unterrichtsorganisation, die Bildung von Lerngruppen nach Bildungsbedarfen etc. erforderlich. Die konsequente Beachtung des Inklusionsgedankens kann aus diesen scheinbaren Notlösungen neue Potenziale entwickeln und die Angebote für die neuen Bildungsbedarfe öffnen. Hier setzten die Verzahnung und Kooperation auf regionaler Ebene und die Angebotsautonomie an. Zugleich ist hier die Verbindung zur Unterrichtsebene und zur Schulkultur gegeben. 


\section{Inklusion als Herausforderung}

Es gibt eine Vielzahl von Veröffentlichungen zur Schulkultur, zur Schulentwicklung, zur Schul- und Unterrichtsqualität sowie zum selbstorganisierten und selbstsorgenden Lernen, zur interkulturellen und zur Förderpädagogik, an welche die inklusive Schulentwicklung, Didaktik und Lerngestaltung anschließen können. Diese können hier jedoch nicht systematisch diskutiert werden. Deshalb wird nur auf einige wenige Studien und Konzepte aufmerksam gemacht.

Als erstes ist auf den „Index für Inklusion“ hinzuweisen (vgl. Boban \& Hinz, 2003). Dieser enthält drei Dimensionen und sechs Bereiche. Diese sind (1) ,,inklusive Strukturen schaffen" mit den Bereichen „Gemeinschaften bilden und inklusive Werte verankern“, (2) „,inklusive Strukturen etablieren“ mit den Bereichen „eine Schule für alle entwickeln und Unterstützung für Vielfalt organisieren" sowie (3) „, inklusive Praktiken entwickeln“ mit den Bereichen „Lernarrangements organisieren und Ressourcen mobilisieren". Weitere 44 Indikatoren dienen der konkreten Verständigung, Entwicklung und insbesondere der Evaluation des erreichten Standes. Mit den in einem Index formulierten 560 Fragen sollen die Barrieren gemeinschaftlich erkannt und in einem gemeinsamen Prozess überwunden werden.

Meyer (2003) und Helmke (2004) haben Kriterien für guten Unterricht herausgearbeitet. Gegenwärtig wird die Diskussion von den Ergebnissen der Hattie-Studie (Hattie, 2008) beherrscht, die eine Bilanz zur Wirksamkeit von Lehren und Lernen aus 800 Metaanalysen zieht und darauf aufbauend eine Konzeptionen für nachhaltiges Lernen und wirksames Lehren entwickelt (Hattie, 2011). Die in diesen Publikationen vorgestellten Kriterien und Prinzipien sind kompatibel mit inklusiver Didaktik und inklusivem Unterricht. Sie sind jedoch noch in inklusive Ansätze und Lernformen aus der Förderpädagogik zu überführen und entsprechend zu modifizieren bzw. zu gewichten, denn erst diese Ansätze orientieren sich explizit an den Prinzipien inklusiver Bildung. Hier ist insbesondere der Ansatz von Feuser ( 2001) zu nennen, zum Thema interkulturelles Lernen vgl. u.a. Auernheimer, 2010, zum Diversity Management vgl. Kimmelmann, 2010 oder zum Anti-Bias-Ansatz vgl. Gramelt, 2010. Vergleichbares gilt für die Professionalität des Bildungspersonals. Ausgearbeitete Analysen liegen hierzu von Bylinski (2013) und Buchmann \& Bylinski (2013) vor.

\section{Literatur}

Ahmadi, S. \& Kolland, F. (2010). Stabilität und Wandel: Bildung im Lebenslauf. Zeitschrift für Weiterbildungsforschung, 33 (3), 43-53.

Aichele, V. (2010). Monitoring - unverzichtbarer Beitrag zur staatlichen Umsetzung der UN-Behindertenrechtskonvention. [www.institut-fuer-menschenrechte.de].

Althusmann, B. (2011). Fachgespräch zur Empfehlung der Kultusministerkonferenz: Inklusive Bildung von Kindern und Jugendlichen mit Behinderungen in Schulen. [www.kmk.org/presse-und-aktuelles/meldung/fachgespraech-zur-empfehlungder-kultusministerkonferenz-inklusive-bildung-von-kindern-und-jugend.html]. 


\section{Inklusion als Herausforderung}

Auernheimer, G. (2010). Interkulturelle Bildung im Jahr 10 des einundzwanzigsten Jahrhunderts. In H.R. Yousefi (Hrsg), Wissensgesellschaft im Wandel. Bildung, Bolognaprozess und Integration in der Diskussion. (S.19-37). Nordhausen: Bautz.

Autorengruppe Bildungsberichterstattung (Hrsg.). (2010). Bildung in Deutschland 2010. Bielefeld.

Autorengruppe Bildungsberichterstattung (Hrsg.). (2012). Bildung in Deutschland 2012. Bielefeld.

Baethge, M. (2012). Demografie als Chance: Perspektiven für die berufliche Bildung. Jahresforum der „Weinheimer Initiative“, Kiel.

[www.sfs.tudortmund.de/v2/dokumente/aktuelles/veranstaltungen/veranstaltungs archiv/Prof._Dr._Martin_Baethge_PraesentationJahresforum_ Kiel_1.2._Februar_2012.pdf].

Boban, I. \& Hinz, A. (Hrsg). (2003). Index für Inklusion. Lernen und Teilhabe in Schulen der Vielfalt entwickeln. Halle: Martin Luther Universität.

Buchmann, U. \& Bylinski, U. (2013). Ausbildung und Professionalisierung von Fachkräften für eine inklusive Berufsbildung. In H. Döbert \& H. Weishaupt (Hrsg.), Inklusive Bildung professionell gestalten. Situationsanalyse und Handlungsempfehlungen (S. 147-202). Münster: Waxmann.

Bundesministerium für Arbeit und Soziales. (2013). Inklusionsinitiative für Ausbildung und Beschäftigung: Neue Chancen für Menschen mit Behinderungen auf dem Arbeitsmarkt.

[www.bmas.de/DE/Themen/Teilhabe-behinderter-Menschen/Meldungen/201310-25-inklusionsinitiative.html]

Bundesministerium für Arbeit und Soziales (2013a). Nationaler Aktionsplan der Bundesregierung zur Umsetzung der UN-Behindertenrechtskonvention. [http://www.bmas.de].

Bylinski, U. (2013). Eine inklusive Berufsbildung braucht die Professionalität des Bildungspersonals. berufsbildung, 66 (143), 23-25.

Bylinski, U. \& Rützel, J. (2011). „Ausbildung für alle“ braucht eine Pädagogik der Vielfalt. $B W P, 40(2), 14 \mathrm{ff}$.

Dammberger, T. (2014). Mensch 2.0 - Erweiterte Bildung? In berufsbildung, (68) 145 (im Druck).

Deller, J., Kern, S., Hausmann, E. \& Dietrichs, Y. (2008). Personalmangagement im Demographischen Wandel: Ein Handbuch für den Veränderungsprozess. Heidelberg: Springer.

Expertengruppe zur Weiterentwicklung der beruflichen Schulen in Rheinland-Pfalz. (2013). Ergebnisse: Internes Papier.

Feuser, G. (2001). Qualitätsmerkmale integrativen Unterricht.

[www.georg-feuser.com/conpresso/_data/Qualitätsmerkmale_integrativen_ Unterrichts_Wien_2001-10-20.pdf]. 


\section{Inklusion als Herausforderung}

Fisseler, B. \& Schaten, M. (2012). Barrierefreies E-Learning in der Berufsbildung. berufsbildung, 66 (137), 21-23.

Gans, S. (2011). Bevölkerung - Entwicklung und Demographie unserer Gesellschaft. Darmstadt: Primus.

Gramelt, K. (2010). Der Anti-Bias-Ansatz: Zu Konzept und Praxis einer Pädagogik für den Umgang mit (kultureller) Vielfalt. Wiesbaden: VS.

Hattie, J. (2008). Visible Learning - a synthesis of over 800 meta-analysis relating to achievement. London, New York: Routledge.

Hattie, J. (2011). Visible Learning for Teachers - maximizing impact of learning. London, New York: Routledge.

Helmke, A. (2004). Unterrichtsqualität erfassen, bewerten, verbessern. Seelze: Kallmeyer.

Internationales Design-Zentrum Berlin (IDZ) (2014). Was bedeutet Universal Design? [www.idz.de/de/sites/1368.html].

Kimmelmann, N. (2010). Cultural Diversity als Herausforderung der beruflichen Bildung: Standards für die Aus- und Weiterbildung von pädagogischen Professionals im Kontext von Diversity Management. Aachen: Shaker.

Klemm, K. \& Preuss-Lausitz, U. (2011). Auf dem Weg zur schulischen Inklusion in Nordrhein-Westfalen: Empfehlungen zur Umsetzung der UN-Behindertenrechtskonvention im Bereich der allgemeinen Schulen.

[www.dgfe.de/fileadmin/OrdnerRedakteure/Sektionen/Sek06_SondPaed/Studie Klemm_Preuss-Lausitz_NRW_Inklusionskonzept_2011.pdf].

Meyer, M. (2003). Zehn Merkmale guten Unterrichts. Pädagogik, 65 (10), 36-43.

Möller, J. (2013). Effekte inklusiver Beschulung aus empirischer Sicht. In J. Baumert (Hrsg.), Inklusion. Schulmanagement Handbuch 146 (S. 15-37). München: Oldenbourg.

Patt, R. (2012). 13 Gütekriterien der inklusiven Konstruktion. In K. Reich (Hrsg.), Inklusion und Bildungsgerechtigkeit. Standards und Regeln zur Umsetzung einer inklusiven Schule (S. 210-216). Weinheim: Beltz.

Reich, K. (Hrsg.). (2012). Inklusion und Bildungsgerechtigkeit: Standards und Regeln zur Umsetzung einer inklusiven Schule. Weinheim: Beltz.

Rützel, J. (2000). Vocational training cooperation in times of internationalisation and individualisation. In G. Kohn, J. Rützel, H. Schröter \& S. Ziehm (Hrsg.), Compatibility of Vocational Qualification Systems (S. 26). Berlin: Overall.

Rützel, J. (2013). Inklusion als Perspektive einer zukunftsorientierten Berufsbildung und die Bewältigung des demographischen Wandels. In D. Münk (Hrsg.), bwp@ Spezial 6 - Hochschultage Berufliche Bildung 2013, Workshop 22 Demographischer Wandel und Fachkräftesicherung im Berufsbildungsraum Rhein-Ruhr. [www.bwpat.de/ht2013/ws22/ruetzel_ws22-ht2013.pdf].

Sarter, M. (2013). Demografie, Fachkräfte, Handlungsfelder.

[http://berufsbildendeschule.bildungrS.de/fileadmin/user_upload/bbs/ 


\section{Inklusion als Herausforderung}

berufsbildendeschule.bildungrS.de/aktuelles_meldungen/sonstige/

LDK_2013_Frau_Martina_Sarter_BA.pdf].

Sicking, S. (2012). Inklusion - ein Beitrag zur Bewältigung des demographischen Wandels. berufsbildung, 66 (137), 4-6.

Statistisches Bundesamt. (2009). Vorausberechnung der Bevölkerungsentwicklung bis 2060. [www.destatis.de/DE/Publikationen/Thematisch/Bevoelkerung/ VorausberechnungBevoelkerung].

Tenorth, H.-E. (2013). Inklusion - Prämissen und Problemzonen eines kontroversen Themas. In J. Baumert (Hrsg.), Inklusion. Schulmanagement Handbuch 146 (S. 6-14). München: Oldenbourg.

Tippelt, R., Schmidt, B., Schnurr, S., Sinner, S. \& Theisen, C. (2009). Bildung Älterer-Chancen im demografischen Wandel. Bielefeld: Bertelsmann.

\section{Verfasser}

Prof. Dr. Josef Rützel

Technische Universität Darmstadt

Institut für Allgemeinte Pädagogik und Berufspädagogik

Alexanderstraße 6

E-Mail: ruetzel@bpaed.tu-darmstadt.de 